\title{
VALORACIÓN ULTRASONOGRÁFICA SIMPLIFICADA DEL OLIGOHIDRAMNIOS E INDICACIÓN DE CESÁREA POR SUFRIMIENTO FETAL
}

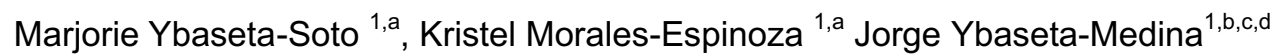

1. Facultad de Medicina, Universidad Nacional San Luís Gonzaga. Ica, Perú

a. Médico Cirujano b. Médico Gineco-Obstetra c. Doctor en Medicina d. Hospital Santa María del Socorro. Ica, Perú RESUMEN

Objetivo: Determinar si el oligohidramnios medido con la técnica Ultrasonográfica simplificada del bolsillo vertical mayor de líquido amniótico tiene relación con la indicación de cesárea por sufrimiento fetal. Material y métodos: Se realizó un estudio observacional, transversal y analítico, se incluyeron gestantes de 37 a 41 semanas en quienes se valoró oligohidramnios mediante la técnica del bolsillo vertical mayor (BVM). Resultados: El grupo de oligohidramnios medido por BVM $\leq 2 \mathrm{~cm}$ presentó frecuencias estadísticamente significativas para trazados cardiotocográficos patológicos $(p=0,002)$, parto por cesárea $(p=000,1)$, liquido amniótico meconial $(p=0,0001)$, score de Apgar menor de siete al minuto $(p=0,03)$ y a los cinco minutos $(p=0,003)$ e ingreso a la unidad de cuidados intensivos neonatales $(p=0,008)$. Conclusión: Se encontró relación entre el oligohidramnios valorado mediante la técnica ultrasonográfica del bolsillo vertical mayor y la indicación de cesárea por sufrimiento fetal. (Rev. méd. panacea 2011; 1:34-36).

Palabras clave: oligohidramnios, cesárea y sufrimiento fetal.

\section{SIMPLIFIED ULTRASONOGRAPHY ASSESSMENT OF OLIGOHYDRAMNIOS AND CESAREAN INDICATION FOR FETAL DISTRESS}

\section{ABSTRACT}

Objetive: To determine if the oligohydramnios measured with ultrasound simplified-technique from largest vertical pocket of amniotic fluid is related to the cesarean indication for fetal distress. Material and Methods: It was realized an observationaltransversal- analytical study, pregnant women from 37 to 41 weeks were included in whom these oligohydramnios was assessed using the technique of largest vertical pocket (LVP). Results: Group measured by LVP oligohydramnios $\leq 2 \mathrm{~cm}$ showed frequencies statistically significant for pathological CTG tracings $(p=0.002)$, cesarean delivery $(p=000.1)$, meconium ( $p=0.0001)$, Apgar score less than seven at one minute $(p=0.03)$ and five minutes $(p=0.003)$ and admission to neonatal intensive care unit $(p=0.008)$. Conclusion: It was found a relation between oligohydramnios assessed by ultrasound technique from largest vertical pocket and indication of cesarean section for fetal distress . (Rev. méd. panacea 2011; 1:34-36).

Key words: oligohydramnios, caesarean and fetal distress.

\section{INTRODUCCIÓN}

La tasa de mortalidad fetal por oligohidramnios es alta alrededor del $80 \%$ y el pronóstico empeora cuando mas temprano se presenta y puede estar asociado a oliguria fetal, retardo del crecimiento intrauterino, postmadurez o disfunción placentaria ${ }^{(1,2)}$. La existencia de disminución del líquido amniótico (LA) es diagnosticado por procedimientos ultrasonográficos adecuados, por lo que se hace evidente la importancia de estas evaluaciones en el control prenatal y en el anteparto ${ }^{(1,2)}$.

El oligohidramnios es la disminución del volumen del líquido amniótico aproximadamente por debajo de $400 \mathrm{ml}$ al término del embarazo, medido ultrasonográficamente por la técnica del BVM es menor a dos centímetros o un índice de líquido amniótico menor o igual a cinco, así como la medida de dos diámetros menor a quince centímetros cuadrados $(1,2)$

En diferentes estudios se ha reportado que un volumen disminuido de líquido amniótico, está asociado a un riesgo significativo de operación cesárea por sufrimiento fetal, presentar Apgar a los 5 minutos menor de 7 y trazados anormales de la frecuencia cardiaca ${ }^{(2,3)}$. Posteriormente, otros investigadores reportaron la relación existente entre el índice de líquido amniótico disminuido y un mayor riesgo de presentar líquido amniótico teñido de meconio, cardiotocografía (CTG) patológica, acidosis fetal, ingreso a la unidad de cuidados intensivos neonatales ${ }^{(4,5)}$.

La presente investigación busca determinar si el oligohidramnios medido con la técnica ultrasonográfica del bolsillo vertical mayor presenta relación con la indicación de cesárea por sufrimiento fetal.

\section{MATERIAL Y MÉTODOS}

Se realizó un estudio observacional de corte transversal analítico.

L a muestra estudiada estuvo constituida por 118 pacientes con gestación única, de 37 a 41 semanas de gestación, presentación fetal de vértex y membranas amnióticas 
intactas; que culminaron su embarazo en el Departamento de Gíneco-Obstetricia del Hospital Santa María del Socorro de Ica, en el período comprendido entre julio- diciembre del año 2010.

Se excluyeron gestantes con parto inminente y antecedente de cesárea anterior, así como a las que presentaron polihidramnios y trazado anormal de la frecuencia cardiaca fetal al momento del ingreso.

El muestreo fue no probabilístico incidental, quedando la muestra conformada por un total de 118 gestantes.

A todas las gestantes del estudio se le valoró la cantidad del líquido amniótico mediante ecografía considerando que este procedimiento debe ser realizado (tener un tiempo de latencia de) una semana antes del parto (en relación al parto). El procedimiento consiste en dividir la cavidad uterina en cuatro cuadrantes, midiendo luego la profundidad vertical del bolsillo mayor libre de cordón y partes fetales. Definiendo como oligohidramnios un valor $\leq 2 \mathrm{~cm}$.

Para recabar la información se utilizó una ficha de recolección de datos elaborada para cumplir los fines del estudio, siendo la fuente el libro de ingresos de sala de labor de parto; así como el libro de registro de pacientes que terminaron en operación cesárea.

Para el análisis se elaboró una base de datos con el programa estadístico Excel, los datos fueron analizados con el programa SPSS 13. Las comparaciones entre variables se presentan en forma de proporciones, las diferencias se midieron aplicando la prueba Chi Cuadrado aceptando como significativo un valor de $p<0,05$ para un intervalo de confianza del $95 \%$. El análisis y los resultados se presentan en tablas de doble entrada

\section{RESULTADOS}

El rango de edades de la población gestante estudiada fue de 15 a 43 años con una media de 24,8 años, la media de edad gestacional al momento de parto fue de 38,9 semanas. Del total de recién nacidos $52(46,6 \%)$ fueron de sexo masculino y $66(55,9 \%)$ femenino.

De las 14 pacientes con Bolsillo Vertical Mayor $2 \mathrm{~cm} \mathrm{(12 \% )}$ la media de edad materna fue de 22,7 años, en el grupo de 104 pacientes con Bolsillo Vertical Mayor $>2 \mathrm{~cm}$ fue de 26,1 años (88\%).

El grupo de oligohidramnios, aquel con Bolsillo Vertical Mayor $\leq 2 \mathrm{~cm}$. (14 pacientes), presentó frecuencias estadísticamente significativas para: trazados cardiotocográficos patológicos $(p=0,002)$, parto por cesárea
( $p=0,0001)$, líquido amniótico meconial $(p=0,0001)$; score de Apgar menor de siete al minuto $(p=0,03)$ y a los cinco minutos $(p=0,003)$ e ingreso a la unidad de cuidados intensivos neonatales $(p=0,008)$ (Tabla 1$)$.

Tabla 1. Bolsillo vertical mayor (BVM) y sufrimiento fetal por oligohidramnios

\begin{tabular}{|c|c|c|c|c|c|}
\hline \multirow{2}{*}{ Variables } & \multirow{2}{*}{$\frac{\text { BVM }}{n}$} & \multirow{2}{*}{$\begin{array}{c}\leq 2 \mathrm{~cm} \\
(\%)\end{array}$} & \multicolumn{2}{|c|}{$\mathrm{BVM}>2 \mathrm{~cm}$} & \multirow{2}{*}{$p$} \\
\hline & & & 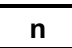 & $(\%)$ & \\
\hline \multicolumn{6}{|l|}{ Cardiotocografía } \\
\hline Normal & 6 & $(42,86)$ & 93 & $(89,42)$ & 0,0002 \\
\hline Patológico & 8 & $(57,14)$ & 11 & $(10,58)$ & \\
\hline \multicolumn{6}{|l|}{ Tipo de parto } \\
\hline Vaginal & 6 & $(42,86)$ & 92 & $(88,46)$ & 0,0001 \\
\hline Cesárea & 8 & $(57,14)$ & 12 & $(11,54)$ & \\
\hline \multicolumn{6}{|c|}{ Color de liquido amniótico } \\
\hline Claro & 4 & $(28,57)$ & 83 & $(79,81)$ & 0,0001 \\
\hline Verde fluido & 2 & $(14,29)$ & 18 & $(17,31)$ & \\
\hline Meconial & 8 & $(57,14)$ & 3 & $(2,88)$ & \\
\hline \multicolumn{6}{|l|}{ Distocia funicular } \\
\hline En parto vaginal & 2 & $(40)$ & 23 & $(76,67)$ & 0,09 \\
\hline En cesáreas & 3 & $(60)$ & 7 & $(23,33)$ & \\
\hline \multicolumn{6}{|l|}{ Apgar al minuto } \\
\hline$<7$ & 3 & $(21,43)$ & 6 & $(5,77)$ & 0,0382 \\
\hline$>7$ & 11 & $(78,57)$ & 98 & $(94,23)$ & \\
\hline \multicolumn{6}{|c|}{ Apgar a los cinco minutos } \\
\hline$<7$ & 3 & $(21,43)$ & 3 & $(2,88)$ & 0,003 \\
\hline$>7$ & 11 & $(78,57)$ & 101 & $(97,12)$ & \\
\hline \multicolumn{6}{|l|}{ Ingreso a UCI } \\
\hline SI & 5 & $(35,71)$ & 7 & $(6,73)$ & 0,0008 \\
\hline No & 9 & $(64,29)$ & 97 & $(93,27)$ & \\
\hline
\end{tabular}

\section{DISCUSIÓN}

La valoración del líquido amniótico mediante la técnica ultrasonográfica del bolsillo vertical mayor ha demostrado ser un parámetro que permite seleccionar las gestantes con mayor riesgo de presentar resultados perinatales adversos cuando este es menor a $2 \mathrm{~cm}^{(6)}$.

Debido a que el diagnóstico de sufrimiento fetal implica presencia de trazados patológicos del ritmo cardiaco fetal en la presente investigación se evidenció que la frecuencia de trazados patológicos cardiotocográficos fue significativamente mayor en el grupo del BVM $2 \mathrm{~cm}$, de igual manera, otros estudios también han reportado cardiotocografías patológicas en relación con la presencia de oligohidramnios ${ }^{(7)}$.

Nuestro resultado es congruente con el publicado por Balestena y $\mathrm{col}^{\left({ }^{(8)}\right.}$ quien refiere que las gestantes con oligohidramnios pueden mostrar signos de sufrimiento fetal y por lo tanto, es necesario hacer de la cesárea la vía más rápida e idónea para el nacimiento. 
El diagnóstico de sufrimiento fetal relacionado con oligohidramnios implica presencia de trazados patológicos del ritmo cardiaco fetal principalmente desaceleraciones variables y esta asociado a resultados adversos como apgar menor a 7 al minuto y a los cinco minutos, líquido amniótico meconial e ingreso a la unidad de cuidados intensivos $(4,5,9,10)$.

En la bibliografía consultada se indica dependencia de asfixia perinatal dada por el parámetro del Apgar, considerando que un producto con oligohidramnios es un factor predictivo para sufrimiento fetal, resultando un recién nacido deprimido que requieren ser admitidos a la unidad de cuidados intensivos ${ }^{(10-14)}$, sin embargo, otros autores no han podido confirmar que la frecuencia de ingresos a UCIN sea significativamente mayor en los neonatos con oligohidramnios ${ }^{(15,16)}$

Pensamos que esta técnica de evaluación del líquido amniótico que es sencilla, rápida y de fácil aplicación, al integrarla en la evaluación del control prenatal nos aportaría un elemento más en la vigilancia de la salud fetal.

Recibido: 20-07-11 Aprobado: 14 -09-11

Financiamiento: El estudio fue autofinanciado

Conflictos de interés: No declarados

1. Huamán M, Sosa $\mathbf{A}$, Pacheco J. Ecografía en Obstetricia, medicina fetal y ginecología 2D/ Doppler/3D-4D. Lima-Perú. Instituto latino americano de salud reproductiva EPUS; 2009. p. 78.

2. Clement D, Schifrin BS, Kates RB. Acute oligohidramnios in post date pregnancy. Am J Obstet Gynecol 1987; 157:884-6.

3. Manning FA, Hill LM, Platt LD. Qualitative amniotic fluid volume determination by ultrasound: antepartum detection of intrauterine growth retardation. Am J Obstet Gynecol 1981;139: 254-8.

4. Brost BC, Scardo JA, Newman RB, Van Dorsten JP. Effect of fetal presentation on the amniotic fluid index. Am J Obstet Gynecol 1999;181:1222-4.

5. Flack NJ, Sepulveda W, Bower S, Fisk NM. Acute maternal hydration in third-trimester oligohydramnios: effects on amniotic fluid volume, uteroplacental perfusion and fetal blood flow and urine output. Am J Obstet Gynecol 1995;173:1186-91.

6. Lagos RA. Valoración ultrasonográfica simplificada del líquido amniótico y su asociación con el crecimiento fetal. Rev chil Ultrasonog 2008; 11:46-52.
7. Rivero MI, Alegre MC, Feu Mc, Gonzalea Ma. Caracterización del sufrimiento fetal agudo en un medio hospitalario. Rev Med Norest 2007; 9:12-18

8. Balastena JM, Almeida G, Balestena S. Resultados del oligohidramnios en el parto y el recién nacido. Rev. Cub Obstet Ginecol 2005, 31(1).

9. Phelan JP, Ahn MO, Smith CV, Rutherford SE, Anderson E. Amniotic fluid index measurements during pregnancy. J Reprod Med 1987;32: 601- 4.

10. Clark SL, Sabey P, Jolley K. Nonstress testing with acoustic stimulation and amniotic fluid volume assessment: 5973 tests without unexpected fetal death. Am J Obstet Gynecol 1989;160:694-7.

11. Lucero F. Pronóstico perinatal en recién nacidos hijos de madres con oligohidramnios y NST reactivo [Tesis Médico Cirujano]. Guatemala: San carlos Univ: 2000.

12. Callen Pw. Ecografía en Obstetricia y ginecología. $5^{a}$ ed. Elsiever-Masson: España, 2009.

13. Nabhan AF, Abdelmoula YA. Amniotic fluid index versus single deepest vertical pocket: a meta-analysis of randomized controlled trials. Int J Gynaecol Obstet. 2009 Mar;104(3):184-8

14. Tharmaratnam S. Fetal distress. Baillieres Best Pract Res Clin Obstet Gynecol 2000; 14(1): 155-72.

15. Clement D, Schifrin BS, Kates RB. Acute oligohidramnios in post date pregnancy. Am J Obstet Gynecol 1987; 157:884-6..

16. Coulson CC, Thorp JM, Purrington J, Slotchiver JM, Anath CV, Hartmann K. Effects of maternal smoking on amniotic fluid volume and fetal urine output. Am J Perinatol 1996;13:195-7.

Correspondencia: Dra. Marjorie Ybaseta Soto

Correo electrónico: marjorie_ybaseta@hotmail.com 